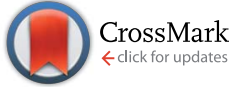

Cite this: RSC Adv., 2017, 7, 7836

Received 12th December 2016 Accepted 17th January 2017

DOI: 10.1039/c6ra28066h

www.rsc.org/advances

\section{Fourier transform ion mobility spectrometry with multinozzle emitter array electrospray ionization}

\author{
Hongling Shen, ${ }^{a}$ Xu Jia, ${ }^{a}$ Qingyan Meng, ${ }^{\text {ab }}$ Wenjie Liu${ }^{\star a b}$ and Herbert H. Hill Jr ${ }^{\mathrm{c}}$
}

Fourier transform ion mobility spectrometry (FT-IMS) is a useful multiplexing method for improving the duty cycle (DC) of IMS from 1 to $25 \%$ when using an entrance and exit ion gate to modulate the ion current with a synchronized square wave chirp. To improve sensitivity further, this paper investigated the performance of FT-IMS using a frequency modulated single gate IMS system coupled to multi emitter array electrospray ionization. Signal to noise ratio, resolving power, linear range and limit of detection were evaluated using various tetra alkyl ammonium salts. The results demonstrated that a significant signal to noise ratio improvement of 4-6 times is achievable with the multi emitter array over that achieved with a single electrospray needle. The limits of detection for various tetraalkylammonium ions declined by 4 times on average and showed improved spectra quality compared to the signal averaging method. Multi emitter array electrospray ionization improved the ion intensities effectively, and Fourier transform IMS improved the duty cycle to $50 \%$ and hence improved the signal to noise ratio further.

\section{Introduction}

Ion mobility spectrometry (IMS) has been recognized as a sensitive, rapid and robust gas phase separation technique and widely applied to the detection of chemical warfare agents (CWA), ${ }^{1,2}$ explosives $^{3-6}$ and illicit drugs. ${ }^{7-9}$ With electrospray ionization (ESI), ion mobility spectrometry is able to detect non-volatile compounds with high sensitivity and accuracy. ESI-IMS has been widely applied to the detection of food contaminants,$^{10}$ pharmaceutics, environmental contaminants, and endogenous physiological compounds..$^{11-13}$

Historically, Fourier transform ion mobility spectrometry (FT-IMS) was the first multiplexing ion mobility method used to improve the IMS duty cycle. ${ }^{14-16}$ By applying a synchronized frequency sweeping square wave to two Bradbury-Nielsen (BN) gates installed at the beginning and the end of the drift region, the duty cycle of FT-IMS was improved to $25 \%$, producing an ion current interferogram. The interferogram could be transformed to an ion mobility spectrum by fast Fourier transform (FFT). Besides the improvement of duty cycle which resulted in a signal to noise ratio enhancement about 3 times, FT-IMS also reduced the peak tailing and baseline shift, thus improving confidence in the identification of the ions. However, when combine electrospray ionization to FT-IMS, only limited signal to noise $(\mathrm{S} / \mathrm{N})$ about 1.4 was achieved which is far lower than

${ }^{a}$ College of Life Science, Tarim University, Alar, Xinjiang, 843300, China. E-mail: lury@sina.com; Tel: +8615569200251

${ }^{b}$ Xinjiang Production \& Construction Corps Key Laboratory of Protection and Utilization of Biological Resources in Tarim Basin, Alar, Xinjiang, 843300, China ${ }^{c}$ Department of Chemistry, Washington State University, Pullman, WA, 99163, USA theoretic estimation. ${ }^{14}$ Another main concern about FT-IMS is the discriminative effect against bigger molecules because of BN gate depletion effect. For traditional FT-IMS uses two ion gate, this doubled the gate depletion effect and hence decreased the ion transmission for low mobility ion species. Another alternative multiplexing technique is Hadamard transform ion mobility spectrometry (HT-IMS). Instead of frequency modulated ion gate, HT-IMS utilizes a binary pseudo random sequence applied to the B-N gate, followed by Hadamard transform to retrieve the deconvoluted IMS spectrum. ${ }^{17}$ Though HT-IMS needs no modification to the IMS hardware and less computationally intensive, the false peaks caused by Hadamard transform are very hard to discriminate from real signal and hence increased the possibility of false alarm and misidentification. ${ }^{18}$

Multinozzle emitter array (MEA) has been applied to the mass spectrometry to improve the ionization efficiency and demonstrate many unique advantages such as the total electrospray current of the multinozzle emitter array is approximately proportional to the square root of the number of nozzles, thus increasing the sensitivity of mass spectrometry. ${ }^{19}$ Another attractive advantage is by reducing the flow rate of individual emitter, MEA showed high tolerance to water content, less ionization fluctuation, and equal mole ionization response to various compounds. ${ }^{20-23}$ The total flow rate of electrospray also improved to several micro liters and reduced the residence time in transfer tubing and improved the post-column performance when coupled to liquid chromatography separations.

For electrospray ionization and traditional drift tube ion mobility spectrometry are both atmosphere technique, thus MEA could improve the performance of ESI-IMS by generating and introducing more ions for separation. ${ }^{24}$ Also, the major 
challenge of MEA coupled to mass spectrometry is the limited transmission efficiency through the atmosphere-vacuum interface. This limitation does not exist for ambient pressure drift tube ion mobility spectrometry. In theory, all ions generated from an MEA could be introduced into an IMS drift tube for separation and detection.

The objective of this paper is to improve the performance of electrospray ionization ion mobility spectrometry by increasing the ion throughput via Fourier transform using the "external" ion gate, and by improving the ionization efficiency of electrospray, which generate more ions through multi emitter array electrospray ionization. The signal to noise ratio, resolving power, limit of detection, dynamic range and calibration curve of both traditional signal average mode and Fourier transform mode are compared and the sampling rate, chirp frequency range, chirp frequency rate and various apodization function are optimized to achieve higher $\mathrm{S} / \mathrm{N}$ ratio.

\section{Experiment section}

\subsection{Instrumental}

The electrospray ionization drift tube ion mobility spectrometer used in this research has been described in detail elsewhere. ${ }^{25,26}$ For electrospray ionization, A $150 \mu \mathrm{m}$ inner diameter silica capillary tube with polyimide coating and a syringe were used for solvent delivery to the ESI needle, ESI needle was a $46 \mu \mathrm{m}$ inner diameter silica capillary tube with polyimide coating. The ESI solvent used in the whole experiment was a solution of $90 \%$ methanol and $10 \%$ water otherwise stated. The solvent flow rate for single ESI was maintained at $2 \mu \mathrm{L} \min ^{-1}$ throughout the experiment. Nitrogen gas was used as both drift gas and sheath gas, at $900 \mathrm{~mL} \mathrm{~min}{ }^{-1}$ and $120 \mathrm{~mL} \mathrm{~min}^{-1}$ separately. A voltage bias of $4.2 \mathrm{kV}$ was applied to the electrospray needle according to the voltage of the first ring of drift tube. For multinozzle emitter array electrospray ionization (multi ESI), $1220 \mu \mathrm{m}$ capillary tubing were arranged as a circular shape with $1.5 \mathrm{~cm}$ ID. ${ }^{24}$ The capillary tubing was inserted to a PEEK tubing and sealed with epoxy adhesive and connect to a zero volume stainless steel connector, and the flow rate was $10 \mu \mathrm{L} \mathrm{min}{ }^{-1}$.

The IMS tube, used in this study was $23.2 \mathrm{~cm}$ in total length, with a $6.4 \mathrm{~cm}$ desolvation region and a $16.8 \mathrm{~cm}$ drift region. The IMS drift tube was maintained at $120{ }^{\circ} \mathrm{C}$ temperature. A Bradbury-Nielsen type ion gate separated the desolvation region and drift region. The ion gate voltage was optimized to cut off the signal to zero while using corona discharge ionization source, which is $\pm 32 \mathrm{~V}$ in this instrument. The first ring of the desolvation region was maintained at a constant electric potential of $10.00 \mathrm{kV}$ and the gate reference voltage was measured 9.02 $\mathrm{kV}$. The stacked stainless steel rings were separated from each other by insulating ceramic rings and were connected externally by resistors creating a potential gradient to guide the ions towards the Faraday plate. A counter current flow of dried nitrogen drift gas was introduced from the back of the Faraday plate to facilitate desolvation of ions and sweeping out any neutral molecules. A Keithley 427 amplifier (Keithley Instruments, Cleveland, $\mathrm{OH}$ ) was utilized for signal amplification. A house made Labview (National Instruments, Austin, TX) based
Table 1 Experimental parameters and conditions

\begin{tabular}{|c|c|}
\hline Experimental parameter & Conditions \\
\hline ESI flow rate & $\begin{array}{l}2 \mu \mathrm{L} \min ^{-1} ; 10 \mu \mathrm{L} \min ^{-1} \\
\text { (MEAs) }\end{array}$ \\
\hline ESI bias & $2.8 \mathrm{kV}, 3.8 \mathrm{kV}$ (MEAs) \\
\hline Drift tube voltage & $10.00 \mathrm{kV}$ \\
\hline Voltage on the ion gate & $9.02 \mathrm{kV}$ \\
\hline Drift gas & Nitrogen \\
\hline Drift gas flow rates & $900 \mathrm{~mL} \mathrm{~min}{ }^{-1}$ \\
\hline Drift tube temperature & $120^{\circ} \mathrm{C}$ \\
\hline $\begin{array}{l}\text { Atmospheric pressure } \\
\text { (Alar, Xinjiang) }\end{array}$ & 728 Torr \\
\hline Gate pulse & $0.2 \mathrm{~ms}$ \\
\hline
\end{tabular}

data acquisition (DAQ) software was used for chirp generation, gate driver control, and fast Fourier transform the interferogram to mobility spectra. The gating electronics is described in detail elsewhere. Two Bertan205B high voltage power supply boxes obtained from Bertan (Hicksville, New York) were used for constant voltage supply to the ESI needle and the first ring of drift tube (Table 1).

\subsection{Chemicals}

A series of tetraalkyimmonium bromide salts (Sigma-Aldrich, St. Louis, MO) were used to evaluate the performance of the FT-IMS technique relative to the signal averaging experiments, including tetrapropylammonium bromide (T3A), tetrapentylammonium bromide (T5A), tetrahexylammoniumbromide (T6A), tetraheptylammonium bromide (T7A), and tetraoctylammonium bromide (T8A).

Methanol was LC-MS grade and obtained from Fisher Scientific (Ottawa, Canada) without any further treatment. Individual $50 \mu \mathrm{M}$ solutions of these salts were made in a $90: 10: 0.1 \%$ mixture of methanol, water, and acetic acid respectively. From these stock solutions a mixture containing all of the quaternary ammonium salts was constructed with concentrations ranging from $\sim 0.5 \mathrm{nM}$ to $50 \mathrm{nM}$. This range was adequate to explore the limit of detection, linear range of the evaluated system on analytes in mixtures of various concentrations. All solutions were infused directly into the electrospray ionization source using a syringe pump (KD Scientific).

\subsection{Data processing}

The identification of ions was performed with reported reduced mobility. Ions in drift tube have a constant drift velocity $(K)$, which proportioned to the electric field $\left(E, \mathrm{~cm}^{2} \mathrm{~s}^{-1}\right)$, in a specific drift gas.

$$
K=\frac{v}{E}=\frac{L^{2}}{V t_{\mathrm{d}}}
$$

where $v$ is the velocity of ion, $L(\mathrm{~cm})$ is the length of the drift region of the drift tube, $V$ is the voltage applied to the ion gate, $t_{\mathrm{d}}$ (s) is the drift time. The variations of temperature and pressure are calibrated with the use of the reduced mobility constant $\left(K_{0}\right.$, $\mathrm{cm}^{2} \mathrm{~V}^{-1} \mathrm{~s}^{-1}$ ) 


$$
K_{0}=K\left(\frac{P}{760}\right)\left(\frac{273}{T}\right)
$$

where $P$ is the atmosphere pressure of the drift tube, $T$ is the temperature of drift gas.

Reduced mobility constant $K_{0}$ of T3A, T5A, T6A, T7A and T8A were calculated with eqn (2), with the results of $1.34,1.00,0.86$, 0.78 and $0.71 \mathrm{~cm}^{2} \mathrm{~V}^{-1} \mathrm{~s}^{-1}$ respectively.

The resolving powers of each compound were calculated with the drift time $\left(t_{\mathrm{d}}\right)$ of the ion divided by the temporal peak width-at-half-height $\left(w_{0.5}\right)$ of the ion mobility peak. This relationship is given by eqn (3):

$$
R_{\mathrm{m}}=\frac{t_{\mathrm{d}}}{w_{0.5}}
$$

where $t_{\mathrm{d}}$ is the drift time of selected ion peak, and $R_{\mathrm{m}}$ is the measured resolving power.

For FT-IMS, the drift times of ions were not measured directly from arrive times of ions. Instead, the calculation of drift time was from time domain peaks transformed from frequency domain interferogram. Because FFT algorithm generates result from each time point of interferogram and expresses with a uniform unit of hertz, the drift time of each peak was calculated by eqn (4):

$$
t_{\mathrm{d}}=\frac{F_{\mathrm{f}} \times T_{\mathrm{s}}}{F_{\mathrm{e}}-F_{0}}
$$

where $t_{\mathrm{d}}$ was the drift time of selected ion peak, $F_{\mathrm{f}}$ was the Fourier transform result from the interferogram, $T_{\mathrm{s}}$ was the scan time, $F_{\mathrm{e}}$ was the ending frequency of ion gate modulation chirp and $F_{0}$ was the starting frequency of chirp signal.

\section{Results and discussion}

\subsection{Comparison between FT transform and SA IMS with single and multi electrospray ionization}

The motivation of multiplexing methods is to increase the ion utilization efficiency and hence improve the signal to noise ratio. For FT-IMS, the duty cycle was improved from typically less than $1 \%$ to constant $50 \%$. To generate a linear frequency sweeping square wave signal for ion gate modulation with $50 \%$ duty cycle, a house made Labview software was developed using the following equation:

$$
x(t)=A \cos \left(2 \pi\left(\frac{k}{2} t+f_{0}\right) t\right)
$$

where $A$ is the voltage to switch the gate controller, $t$ is the time in $\mathrm{ms}, f_{0}$ is the starting frequency, and $k$ is the chirp sweeping rate.

The generated sinusoid frequency sweeping signal was then converted to square wave signal by assign all positive values of eqn (5) to positive ones and all negative values to zeros using a sign function. For visual reason only the first $200 \mathrm{~ms}$ of a spectrum last for $1000 \mathrm{~ms}$ was shown in Fig. 1. This square wave was then sent to the gate controller to switch the ion gate and obtain a raw data which contain the frequency information via NI data acquisition board. The raw data is then multiplied with the square waveform to obtain the time domain

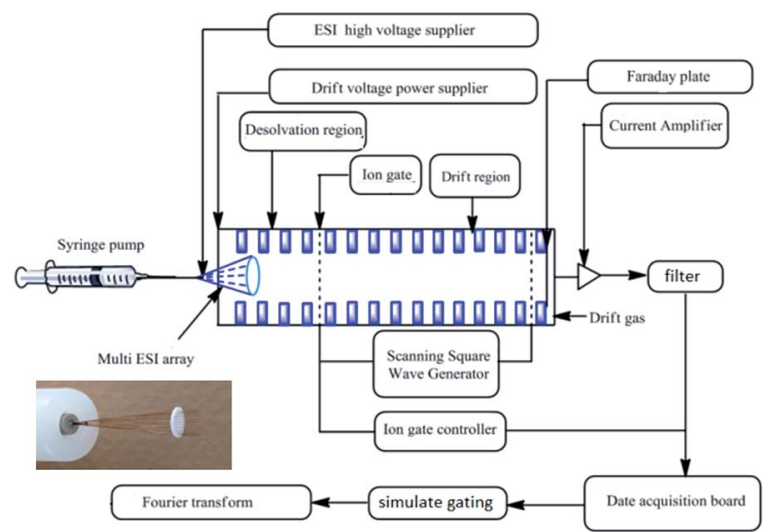

Fig. 1 Diagram of atmosphere pressure Fourier transform multi electrospray ionization ion mobility spectrometer.

interferogram. To avoid baseline distortion because of "frequency leakage", a window function is used to apodize the beginning and ending part of interferogram to zero, then the truncated interferogram is transformed to frequency domain ion mobility spectra by fast Fourier transform (Fig. 2).

Fig. 3 shows ion mobility spectra from signal average mode and Fourier transform when using both single ESI and multi ESI. All four spectra are recorded with the same acquisition time. For signal average mode, the optimum flow rate of ESI solvent was screened using a $90: 10$ methanol to water with $0.1 \%$ glacial acetic acid to obtain a stable ion mobility spectrum. Though we use a relative high drift tube temperature, the limited desolvation capability decreased the performance of ion mobility resolving power for solvent peaks when ESI flow rate increased to $2 \mu \mathrm{L} \mathrm{min}{ }^{-1}$. There is an obvious tailing for solvent peaks under signal averaging mode. Multi ESI using a flow rate

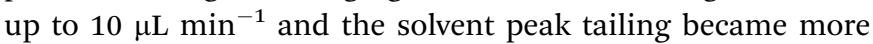
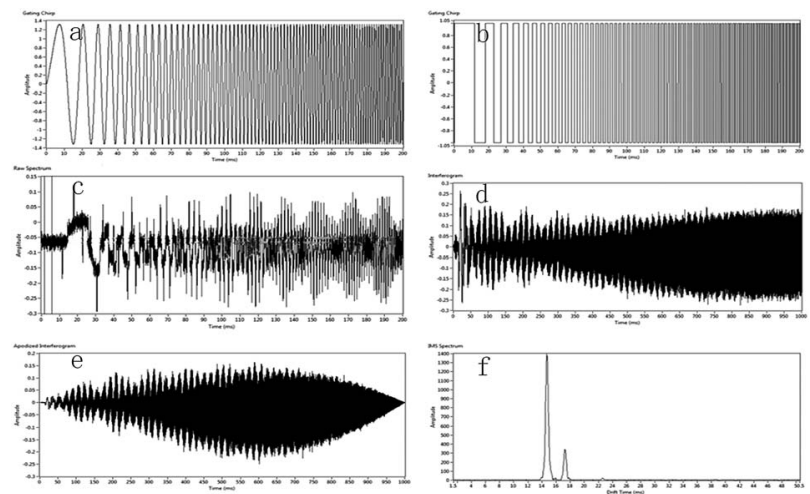

Fig. 2 Generation of gate control square wave frequency sweeping signal and Fourier transform of interferogram to ion mobility spectrum. (a) A sinusoid frequency sweeping waveform start from $20 \mathrm{~Hz}$ to $5000 \mathrm{~Hz}$ within 1 second. (b) Gate control square wave modulation waveform from sinusoid signal using a sign function. (c) The first 200 ms raw data for a frequency modulated ion intensity signal. (d) Interferogram obtained by multiplying square waveform chirp with raw data point by point. (e) Apodizedinterferogram after applying a Welch window function. (f) Ion mobility spectrum from apodized interferogram using a fast Fourier transform. 

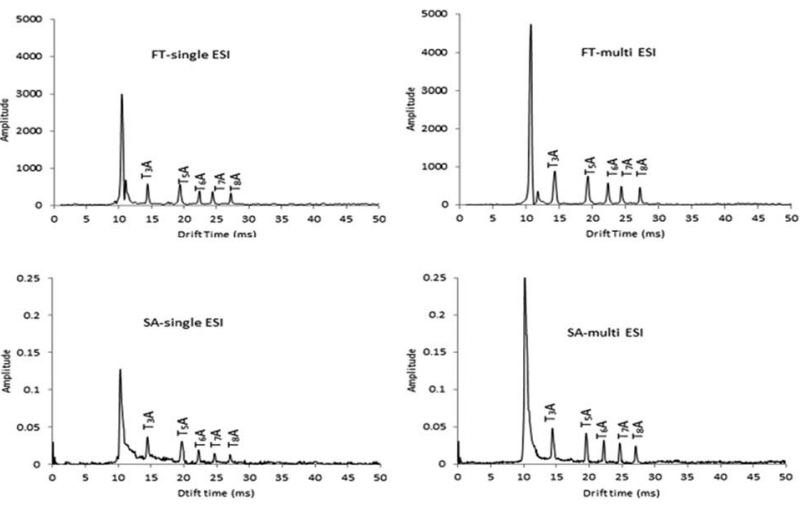

Fig. 3 Typical ion mobility spectra from single ESI signal average (SA) mode; single ESI FT mode; multi ESI SA mode, multi ESI FT mode for T3A, T5A, T6A, T7A and T8A mixed standards.

serious for insufficient desolvation. For Fourier transform IMS only responds to ions resonance between ion gate and detector with characteristic sweeping frequency, those tailing ions decompose in drift tube and exhibit variable drift time are discriminated to form a mobility peak and only contributed to noise. The solvent peaks under FT-IMS with both single ESI and multi ESI show improved resolving power compared to signal averaging mode respectively. For other peaks, the resolving power of FT-IMS are decreased about 10-15\% compared to those of SA mode. One possible reason for this decreased resolving power is to suppress the "frequency leakage" for Fourier transform, both low frequency component and high frequency component of ion interferogram are curtailed using window function and thus decreased the high frequency end useful signal. Another reason is the drift time delay between the gating signal and detected signal, which means the high frequency modulated ion signal aren't detected by the IMS detector and decreased the resolving power of FT mode slightly. Table 2 lists a comprehensive comparison between SA mode

Table 2 Resolving power of five investigated compounds using FT and SA multi-electrospray ionization and single nozzle electrospray ionization source

\begin{tabular}{llll}
\hline $\begin{array}{l}\text { Ionization } \\
\text { source }\end{array}$ & $\begin{array}{l}\text { Mobility } \\
\text { peaks }\end{array}$ & Fourier transform & Signal averaging \\
\hline \multirow{2}{*}{ Multi ESI } & $\begin{array}{l}\text { Solvent } \\
\text { peak }\end{array}$ & $24.39 \pm 0.42$ & $21.10 \pm 0.68$ \\
& T3A & $34.73 \pm 0.42$ & $44.26 \pm 1.97$ \\
& T5A & $49.74 \pm 0.39$ & $63.44 \pm 0.37$ \\
Single ESI & T6A & $64.06 \pm 1.60$ & $71.97 \pm 1.03$ \\
& T7A & $70.446 \pm 1.60$ & $73.78 \pm 0.93$ \\
& T8A & $79.69 \pm 0.29$ & $82.31 \pm 0.35$ \\
& Polvent & $25.22 \pm 0.44$ & $22.93 \pm 0.69$ \\
& T3A & $54.01 \pm 0.95$ & $56.23 \pm 1.52$ \\
& T5A & $57.60 \pm 1.26$ & $65.67 \pm 1.54$ \\
& T6A & $61.67 \pm 0.73$ & $79.35 \pm 0.97$ \\
& T7A & $71.08 \pm 1.26$ & $86.41 \pm 1.19$ \\
& T8A & $90.06 \pm 0.32$ & $97.05 \pm 0.89$
\end{tabular}

and FT mode using single ESI and multi ESI. In all experiments, the acquisition time are set to 2 seconds. Signal averaging using a $0.2 \mathrm{~ms}$ gate pulse width. Correspondingly, FT mode using a maximum frequency of $5 \mathrm{kHz}$, producing a minimum gate pulse of $0.2 \mathrm{~ms}$. Multi ESI also showed slightly decreased resolving power compared to single ESI, this could attribute to improved ion density results in higher ion-ion repulsion and extra peak broadening due to charge-charge repulsion.

\subsection{Sample rate}

The data acquisition rate for Fourier transform is highly depend on the hardware performance including the $10-90 \%$ rise time of current amplifier, resolution and speed of the DAQ board analog to digital converter. In our system, a National Instrument NI PCI 6110 with 12 bits A/D resolution was functioned for analog to digital conversion and data acquisition. Though the maximum sampling rate is up to $1 \mathrm{MS} \mathrm{s}^{-1}$, the noise level increases significantly when it exceeds $50 \mathrm{KS} \mathrm{s}^{-1}$ for reduced A/D conversion time. Fig. 4 compares signal to noise ratio for single ESI and multi ESI ion mobility spectrometry. For various tetraalkylammonium ions, the $\mathrm{S} / \mathrm{N}$-sampling rate relationships demonstrate similar trends for both multi ESI and single ESI, which $\mathrm{S} / \mathrm{N}$ increase along with increased sampling rate, and reach a maximum $\mathrm{S} / \mathrm{N}$ with $50 \mathrm{KS} \mathrm{s}^{-1}$, higher sampling rate more than 50k bring more conversion noise to the spectra and thus signal to noise ratio decreases significantly. It could be expected with higher $\mathrm{A} / \mathrm{D}$ resolution and higher maximum sampling rate DAQ board, the optimum sampling rate could be higher and benefit to track fast ion current change during the experiment.

\subsection{Impact of chirp sweeping time and chirp sweeping rate}

Historically, the Fourier transform ion mobility spectrometry was applied as a highly sensitive and selective gas chromatography detector; however, the traditional two-gate Fourier transform IMS requires relative long sweeping time and hence limited the applications of this operation mode in chromatography detection. To explore the possibility of multi ESI FT-IMS as a HPLC detector, we evaluated the impact of sweeping time and sweeping rate to the performance of detection. Fig. 5 calculated the signal to noise ratio for T3A, T5A, T6A, T7A and

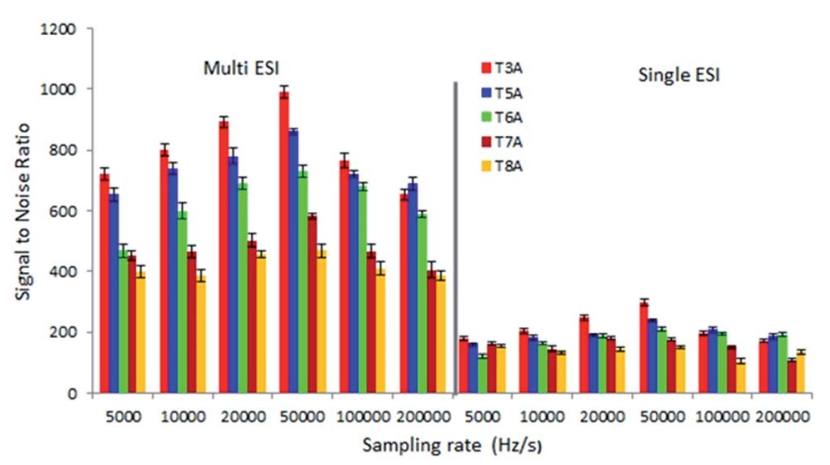

Fig. 4 The influence of sampling rate to Fourier transform ion mobility spectrometry using multi ESI and single ESI. 


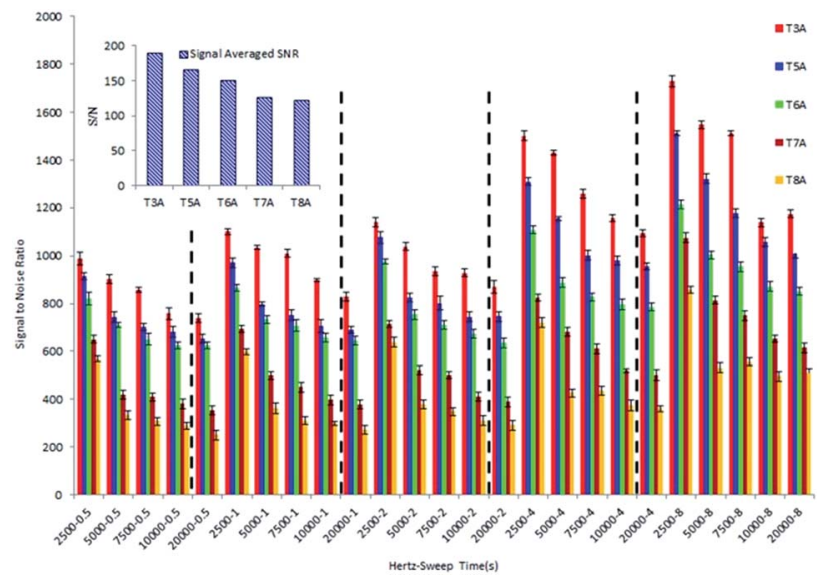

Fig. 5 Multi ESI ion source - impacts of different sweep times on SNR FTIMS.

T8A under FT mode and SA mode. For the same data acquisition time, signal to noise ratio decreases with the increasing of sweeping rate from $2.5 \mathrm{k} \mathrm{Hz} \mathrm{s}^{-1}$ to $20 \mathrm{k} \mathrm{Hz} \mathrm{s}^{-1}$, this is attributed to the non-linear ion transmission over the $\mathrm{B}-\mathrm{N}$ gate for gate depletion. At higher gating frequency, fewer ions could pass through the ion gate during opening period for continues ion beam is eliminated at the gate wires during the closing period to form a depletion zone. However, the resolving power of FT-IMS is directly related to the chirp sweeping rate, thus the compromise between signal to noise ratio and resolving power still need to be made to determine an optimum condition. Longer acquisition time results higher signal to noise ratio with the same sweeping rate. For example, the $\mathrm{S} / \mathrm{N}$ for T5A under $5.0 \mathrm{k} \mathrm{Hz}$ $\mathrm{s}^{-1}$ with 1 second is 820.5 , while 8 seconds acquisition time with the same sweeping rate the $\mathrm{S} / \mathrm{N}$ increased to 1320 . The $\mathrm{S} / \mathrm{N}$ under signal averaging mode is 292.5 instead, the signal to noise ratio improved 4 times approximately. For only one B-N gate is required using this method, the $\mathrm{S} / \mathrm{N}$ gain is higher than previous report. ${ }^{14}$

\subsection{Window function}

Apodization plays a very important role to the quality of IMS spectra quality, especially to the correction of baseline distortion. Commonly chosen window function to apodize the interferogram are Hann function, Hamm function and Gaussian function. However, while with improved spectra quality such as smoother baseline and less peak distortion, the apodization with different window functions also truncate useful signal which decreases the actual signal to noise gain of frequency modulation method. When applied to drift tube ion mobility time of flight mass spectrometry and ion trap mass spectrometry, a two phase out of 180 degree approach was proposed to increase the signal to noise ratio and to diminish the necessity for apodization. With two phase correction, the signal to noise ratio improved significantly for Fourier transform of mass selected interferogram. ${ }^{27}$ However, in our situation, two phase approach is insufficient to suppress the baseline shift alone though some transform artifacts are diminished effectively. To

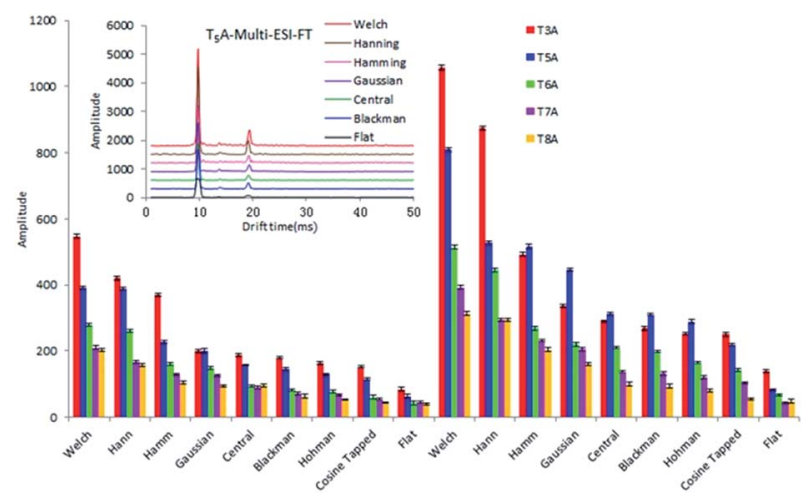

Fig. 6 Impacts of different window functions on the responsive intensity of FTIMS.

improve the signal to noise ratio, useful ion signals should be retained as much as possible before Fourier transform. A detailed signal to noise ratio comparison was performed with various window functions, including Welch function, Hann function, Hamm function and Gaussian function using T3A, T4A, T5A, T6A and T8A (Fig. 6). For all ions compared, Welch function afforded highest signal to noise ratio. Another important concern about window function is the resolving power. Because of resolving power of FT-IMS is determined by the highest frequency of chirp signal applied to the B-N gate, thus higher frequency signal is necessary to obtain high resolving power. Again, Welch function provided the highest resolving power using the same data set. A direct comparison of spectra is listed in the insert of Fig. 6.

\subsection{Quantitative results (LOD, linear range, calibration curve)}

Instead of measuring arrive time of an ion packet, the Fourier transform ion mobility spectrometry measuring the resonance frequency and resonance amplitude of ion current between two gates. Though the improvement of duty cycle by this frequency modulation is constantly improved to $50 \%$ and demonstrated with increased $\mathrm{S} / \mathrm{N}$, the quantitative performance of this

Table 3 Limit of detection comparison between Fourier transform IMS and signal average IMS using multi ESI and single ESI

\begin{tabular}{|c|c|c|c|c|c|}
\hline \multirow[b]{2}{*}{ Ionization } & \multirow[b]{2}{*}{ Compound } & \multicolumn{2}{|l|}{ LOD (FT) } & \multicolumn{2}{|l|}{ LOD (SA) } \\
\hline & & $\mathrm{nmol} \mathrm{mL} \mathrm{m}^{-1}$ & $\mu \mathrm{g} \mathrm{mL} L^{-1}$ & nmol $\mathrm{mL}^{-1}$ & $\mu \mathrm{g} \mathrm{mL} L^{-1}$ \\
\hline \multirow[t]{5}{*}{ Multi-ESI } & T3A & 0.0838 & 0.0223 & 0.2486 & 0.0662 \\
\hline & T5A & 0.0996 & 0.0376 & 0.2657 & 0.1006 \\
\hline & T6A & 0.1282 & 0.0557 & 0.3742 & 0.1626 \\
\hline & T7A & 0.1509 & 0.0745 & 0.4115 & 0.2019 \\
\hline & T8A & 0.1602 & 0.0875 & 0.4774 & 0.2611 \\
\hline \multirow[t]{5}{*}{ Single ESI } & T3A & 0.1301 & 0.0346 & 0.3253 & 0.0865 \\
\hline & $\mathrm{T} 5 \mathrm{~A}$ & 0.1329 & 0.0503 & 0.3921 & 0.1484 \\
\hline & T6A & 0.1638 & 0.0842 & 0.4716 & 0.2049 \\
\hline & T7A & 0.2032 & 0.0997 & 0.5619 & 0.2757 \\
\hline & T8A & 0.2267 & 0.1243 & 0.6516 & 0.3563 \\
\hline
\end{tabular}


Table 4 Analytical parameters for two kinds of compounds using FT and SA nano-electrospray array ionization and single nozzle array electrospray ionization source ${ }^{a, b}$

\begin{tabular}{|c|c|c|c|c|c|c|c|}
\hline ESI & Compounds & Linear equations & $R^{2}$ & $\begin{array}{l}\text { Linear range } \\
\left(\mu \mathrm{mol} \mathrm{mL}^{-1}\right)\end{array}$ & Linear equations & $R^{2}$ & $\begin{array}{l}\text { Linear range } \\
\left(\mu \mathrm{mol} \mathrm{mL}^{-1}\right)\end{array}$ \\
\hline \multirow[t]{3}{*}{ Multi-ESI } & T3A & $y=71.04 x+107.79$ & 0.9973 & $0.156-25$ & $y=0.0064 x+0.0176$ & 0.9981 & $0.312-25$ \\
\hline & T5A & $y=63.23 x+114.53$ & 0.9985 & $0.156-25$ & $y=0.0045 x+0.0178$ & 0.9961 & $0.312-25$ \\
\hline & T6A & $y=46.51 x+55.86$ & 0.9968 & $0.312-25$ & $y=0.0044 x+0.0118$ & 0.9988 & $0.625-25$ \\
\hline \multirow[t]{5}{*}{ Single ESI } & $\mathrm{T} 3 \mathrm{~A}$ & $y=54.57 x+8.84$ & 0.9957 & $0.312-50$ & $y=0.0042 x+0.0148$ & 0.9975 & $0.625-50$ \\
\hline & T5A & $y=51.57 x+51.33$ & 0.9970 & $0.312-50$ & $y=0.0034 x+0.0125$ & 0.9958 & $0.625-50$ \\
\hline & T6A & $y=32.43 x+27.59$ & 0.9965 & $0.312-50$ & $y=0.0032+0.0057$ & 0.9960 & $0.625-50$ \\
\hline & $\mathrm{T} 7 \mathrm{~A}$ & $y=21.32 x+24.09$ & 0.9957 & $0.312-50$ & $y=0.0026 x+0.0050$ & 0.9963 & $0.625-50$ \\
\hline & T8A & $y=17.10 x+15.73$ & 0.9975 & $0.312-50$ & $y=0.0024 x+0.0030$ & 0.9952 & $0.625-50$ \\
\hline
\end{tabular}

${ }^{a} y$ : peak high; $x$ : sample concentration, $\mu \mathrm{mol} \mathrm{mL}{ }^{-1} ; R^{2}$ : squared correlation coefficient. ${ }^{b}$ FT: Fourier transform mode SA: signal-averaging mode.

method is still remain unexplored. To evaluate the quantitative benefit of Fourier transform, the merit of analytic method including linear range, limit of detection and correlation coefficient are compared between signal average method using singular ESI and Fourier transform IMS using multi ESI.

The calibration curves of 5 tetraalkylammonium ions were obtained with six mixed standard solutions of T3A, T5A, T6A, T7A and T8A ranging from 0.5 to $50 \mathrm{nmol} \mathrm{L}^{-1}$, diluted in $90: 10$ ESI solvent with $0.1 \%$ acetic acid. For signal average mode, each spectrum was averaged from 10 iterations of work cycle, and each work cycle were the averaging of 20 single spectrum scan. For FT-IMS, the acquisition time was set to match the signal averaging. The calibration curves for all 5 TXAs showed good linear relationship between the analyte concentration and IMS response, over the concentration range studied with an instrumental detection limit of 0.0223, 0.0376, 0.0557, 0.0745, and $0.0875 \mu \mathrm{g} \mathrm{mL}^{-1}$ for TxAs studied for FT-IMS using multi ESI. For signal average mode using single ESI, the corresponding limit of detection are $0.1301,0.1329,0.1638,0.2032$ and 0.2267 respectively (Table 3). The limit of detection for each ion is calculated at three times the signal to chemical noise ratio. The calibration curves showed correlation coefficient of 0.9973 , 0.9985, 0.9968, 0.9979 and 0.9984 for T3A, T5A, T6A, T7A and T8A using multi ESI FT-IMS respectively. Each point on the calibration curve is the average result of three replicate measurements. There are no significant differences in correlation coefficient between FT and SA mode (Table 4).

\section{Conclusions}

By taking advantages of both Fourier transform multiplexing method and multinozzole emitter array electrospray ionization, we demonstrate a significant sensitivity improve of up to 6 times for drift tube electrospray ionization ion mobility spectrometry. Multi capillary emitter array with a circular configuration to mitigate the field shielding effect and form a homogeneous electrospray plume, and all ions generated from the emitter array are introduced to the drift tube for separation. The limits of detection for various tetraalkylammonium ions are lowed by 4 times and show improved spectra quality compared to signal averaging method. Fourier transform IMS improves the duty cycle to $50 \%$ and hence improves the signal to noise ratio further. The sweeping time could be effectively decreased to 1 second and hence FT-IMS integrated with multi ESI have the potential to serve as a detection method for HPLC separation for improving the signal to noise ratio and solvent flow rate at the same time.

\section{Acknowledgements}

This paper was financially supported by National Science Foundation of China (NSFC 21365018), International Science \& Technology Cooperation Program of XPCC (2015AH005) and Innovation Program of Graduate (TDGRI201523).

\section{References}

1 H. H. Hill and S. J. Martin, Pure Appl. Chem., 2002, 74, 22812291.

2 A. B. Kanu, P. Dwivedi, M. Tam, L. Matz and H. H. Hill, J. Mass Spectrom., 2008, 43, 1-22.

3 R. G. Ewing, D. A. Atkinson, G. A. Eiceman and G. J. Ewing, Talanta, 2001, 54, 515-529.

4 D. S. Moore, Rev. Sci. Instrum., 2004, 75, 2499-2512.

5 J. I. Steinfeld and J. Wormhoudt, Annu. Rev. Phys. Chem., 1998, 49, 203-232.

6 J. Yinon, TrAC, Trends Anal. Chem., 2002, 21, 292-301.

7 S. Armenta, M. Alcala and M. Blanco, Anal. Chim. Acta, 2011, 703, 114-123.

8 T. Keller, A. Keller, E. Tutsch-Bauer and F. Monticelli, Forensic Sci. Int., 2006, 161, 130-140.

9 R. M. O'Donnell, X. B. Sun and P. D. Harrington, TrAC, Trends Anal. Chem., 2008, 27, 44-53.

10 B. Rezaei, M. T. Jafari and O. Rahmanian, Talanta, 2011, 83, 765-769. 
11 G. R. Asbury, J. Klasmeier and H. H. Hill, Talanta, 2000, 50, 1291-1298.

12 H. Borsdorf and G. A. Eiceman, Appl. Spectrosc. Rev., 2006, 41, 323-375.

13 C. Wu, W. F. Siems, J. Klasmeier and H. H. Hill, Anal. Chem., 2000, 72, 391-395.

14 Y. H. Chen, W. F. Siems and H. H. Hill, Anal. Chim. Acta, 1996, 334, 75-84.

15 F. J. Knorr, R. L. Eatherton, W. F. Siems and H. H. Hill, Anal. Chem., 1985, 57, 402-406.

16 E. E. Tarver, Sensors, 2004, 4, 1-13.

17 B. H. Clowers, W. F. Siems, H. H. Hill and S. M. Massick, Anal. Chem., 2006, 78, 44-51.

18 H. Yan, W. Niu, H. Gao, L. Xia, C. Huang and C. Y. Shen, RSC Adv., 2015, 5, 56103-56109.

19 P. Mao, H. T. Wang, P. D. Yang and D. J. Wang, Anal. Chem., 2011, 83, 6082-6089.
20 R. T. Kelly, J. S. Page, I. Marginean, K. Q. Tang and R. D. Smith, Anal. Chem., 2008, 80, 5660-5665.

21 R. T. Kelly, J. S. Page, K. Q. Tang and R. D. Smith, Anal. Chem., 2007, 79, 4192-4198.

22 R. T. Kelly, J. S. Page, R. Zhao, W. J. Qian, H. M. Mottaz, K. Q. Tang and R. D. Smith, Anal. Chem., 2008, 80, 143-149.

23 A. K. Sen, J. Darabi and D. R. Knapp, Microfluid. Nanofluid., 2007, 3, 283-298.

24 W. J. Liu, G. Z. Li, B. Luo, Q. Y. Meng, Y. L. Lu and D. L. Ying, Chin. J. Anal. Chem., 2015, 43, 788-793.

25 D. Wittmer, B. K. Luckenbill, H. H. Hill and Y. H. Chen, Anal. Chem., 1994, 66, 2348-2355.

26 Y. H. Chen, H. H. Hill and D. P. Wittmer, Int. J. Mass Spectrom. Ion Processes, 1996, 154, 1-13.

27 B. H. Clowers, W. F. Siems, Z. H. Yu and A. L. Davis, Analyst, 2015, 14, 6862-6870. 